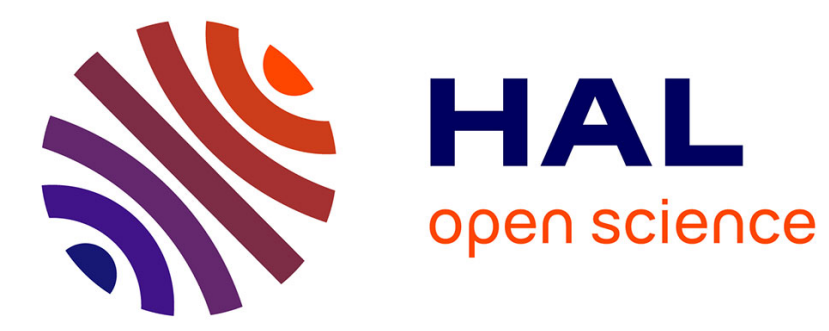

\title{
Decentralized Traffic Management: A Synchronization-Based Intersection Control
}

Mohamed Tlig, Olivier Buffet, Olivier Simonin

\section{To cite this version:}

Mohamed Tlig, Olivier Buffet, Olivier Simonin. Decentralized Traffic Management: A Synchronization-Based Intersection Control. International Conference on Advanced Logistics and Transport, May 2014, Hammamet, Tunisia. hal-00966575

\section{HAL Id: hal-00966575 \\ https://inria.hal.science/hal-00966575}

Submitted on 26 Jun 2014

HAL is a multi-disciplinary open access archive for the deposit and dissemination of scientific research documents, whether they are published or not. The documents may come from teaching and research institutions in France or abroad, or from public or private research centers.
L'archive ouverte pluridisciplinaire HAL, est destinée au dépôt et à la diffusion de documents scientifiques de niveau recherche, publiés ou non, émanant des établissements d'enseignement et de recherche français ou étrangers, des laboratoires publics ou privés. 


\title{
Decentralized Traffic Management: A Synchronization-Based Intersection Control
}

\author{
Mohamed Tlig ${ }^{\dagger *}$, Olivier Buffet* ${ }^{* \dagger}$, Olivier Simonin ${ }^{\ddagger}$ \\ Université de Lorraine ${ }^{\dagger}$ / INRIA Nancy, France* \\ Email: firstname.lastname@loria.fr \\ CITI-INRIA, INSA de Lyon, France $\ddagger$ \\ Email: firstname.lastname@insa-lyon.fr
}

\begin{abstract}
Controlling the vehicle traffic in large networks remains an important challenge in urban environments and transportation systems. Autonomous vehicles are today considered as a promising approach to deal with traffic control. In this paper, we propose a synchronization-based intersection control mechanism to allow the autonomous vehicle-agents to cross without stopping, i.e., in order to avoid congestions (delays) and energy loss. We decentralize the problem by managing the traffic of each intersection independently from others. We define control agents which are able to synchronize the multiple flows of vehicles in each intersection, by alternating vehicles from both directions. We present experimental results in simulation, which allow to evaluate the approach and to compare it with a traffic light strategy. These results show the important gain in terms of time and energy at an intersection and in a network.
\end{abstract}

Keywords-Multi-Agent Systems, Vehicle flow synchronization, Autonomous Vehicles, Traffic Simulations

\section{INTRODUCTION}

In many real transport systems, congestions are generated at the intersections between the roads [1], i.e., parts of the space which must be shared by the vehicles. There exist several methods to manage intersections. The simplest ones generally favor one flow against the other, as traffic lights and "STOP" signals do. Such events generate delays for the vehicles because they require stopping multiple vehicles for some time [2]. If the flow of vehicles is important, these local delays can lead to the emergence of congestions.

This work has been conducted in the context of the InTraDE european project, in which autonomous vehicles transport containers across a seaport ${ }^{1}$. Yet, we consider generic road networks with multiple intersections. Each vehicle follows a pre-determined path along one lane, without turning (changes of direction are not treated here). The objective is to reduce delays and energy consumption, and more generally avoid blockings.

Our approach consists in synchronizing the flows so that the vehicles can alternately cross the intersection without stopping. This requires (1) adapting the vehicles' speeds so that the vehicles arrive at the right time to cross the next intersection without collision, and (2) introducing autonomous control agents at each intersection to handle incoming vehicles. We show how to derive the algorithm in each such control agent and the speed profile for each vehicle as a function of the

\footnotetext{
${ }^{1}$ http://www.intrade-nwe.eu
}

parameters of the problem (spatial dimensions, default speed, angle between roads...).

We empirically evaluate this approach, taking into account various parameters which come into play, such as the throughput of vehicles or the range of the control agent. These experiments are also a means to show how it compares to other approaches. To that end, we consider two metrics: the total delay accumulated by the vehicles while crossing the intersections, and the energy consumption due to the speed variations.

This paper is organized as follows. The next section presents the problem of intersections in general, and existing work. In Section III we present the principle of the temporal synchronization of two roads at an intersection, then explain (i) how to compute the minimum time between two vehicles, and (ii) the algorithm used by the control agent. Section IV is devoted to the experimental study -through simulations- of our model compared to a traditional traffic lights solution. Finally, we discuss the perspectives of this work in the conclusion.

\section{RELATED WORK}

In this study, we address the general problem of managing crossing flows of vehicles in road networks. This problem has been traditionally studied in operations research and queueing theory. It typically concerns vehicles driven by humans but, with the arrival of new technologies, many works integrating on-line decisions consider an automatic and real-time control. Several approaches based on communications and GPS (Global Positioning System) propose to improve existing solutions such as traffic lights. In [3], the authors propose a new strategy to improve traveling times of public transports. The system is based on booking phases of green light at every intersection and gives priority to buses that are furthest behind. In the same context, the authors of [4] propose a strategy that gives priority to buses calculated based on the progress of each of them (along its route) and also on the progress of the following bus.

Other solutions are interested in fully autonomous vehicle control. They can be classified into two categories.

Reservation approaches, introduced by Dresner and Stone in [1], [5], are based on an agent that manages an intersection. Each vehicle wanting to cross must book a passage time interval and a route. The advantage of this approach is that, if several vehicles want to pass and if their paths through the junction do not intersect, then all of them can be satisfied. But, 
otherwise, it is necessary to give priority to one vehicle over the others.

The decentralized approach introduced by Rashe and Naumann [6], [7] is based mainly on communication and negotiation between the vehicles to determine the sequence of passage and exit from the intersection. This approach is known for its limits, which depend on the number of vehicles trying to negotiate their passage through the intersection.

\section{INTERSECTION SYNCHRONIZATION}

\section{A. Local Synchronization Approach}

In this section we define our approach to synchronize the crossing of vehicle flows at an intersection. Before describing how it works, we present the type of road network we are working with. We consider road networks made of roads -with one lane or two opposite lanes- and their intersections. The intersections (described by Figure 1) allow crossing a road but not turning. Roads can intersect at any angle (we first consider the case when $\theta=90^{\circ}$, then we generalize).

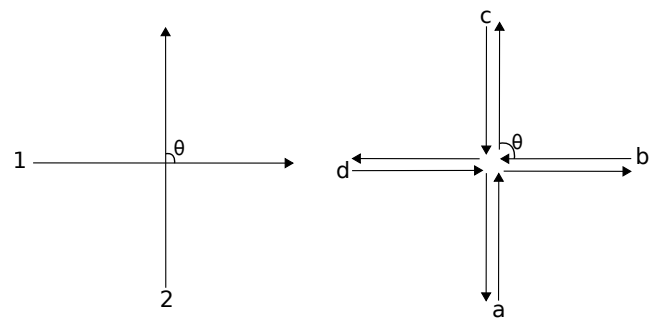

Fig. 1: Intersections with 2 flows (left) and 4 flows (right)

Our approach consists in passing alternately -and without stopping- the vehicles of each road with a sufficient interdistance in order to avoid collisions.

For this purpose, each intersection is handled by a control agent. Its main role is to communicate with the vehicles to manage their passage, allowing them to regulate their speed so as to arrive at the intersection at the right time. The agent has a limited communication range, which defines the distance from which it can start interacting with vehicles approaching the intersection.

The following section explains in more details:

- $\quad$ how to determine the period at which the alternation between roads will take place;

- how a control agent, given this period, controls the vehicles close to its intersection.

\section{B. Minimum Time Period for Crossing}

In this paper, all the vehicles are autonomous and identical. They have a width $w$, a length $l$ and a default speed $V$. The flows are also assumed to be roughly equal on all the lanes of the network.

At first, we focus on the synchronization of two flows $A$ and $B$ to allow their crossing without stopping. We seek to determine the minimum time between two vehicles from the flow $A$, denoted by $T$, that allows passing one vehicle from flow $B$ between them. This means that, on each lane, the average period between two consecutive vehicles should be at least $T$. We distinguish three cases depending on the crossing angle $\theta$ between the two roads: $\theta=90^{\circ}, \theta<90^{\circ}$ and $\theta>90^{\circ}$.

1) $\theta=90^{\circ}$ : The crossing zone is defined by the square corresponding to the space shared by the two roads (see Figure 2). The time required for a vehicle to cross and leave completely the crossing zone is $(l+w) / V$. We deduce the minimum period for passing two vehicles (one from each flow):

$$
T=\frac{2(l+w)}{V},
$$

and the inter-distance between two vehicles from the same flow is $D_{\min }=T * V-l=2(l+w)-l=l+2 w$ (subtracting the length of a vehicle).

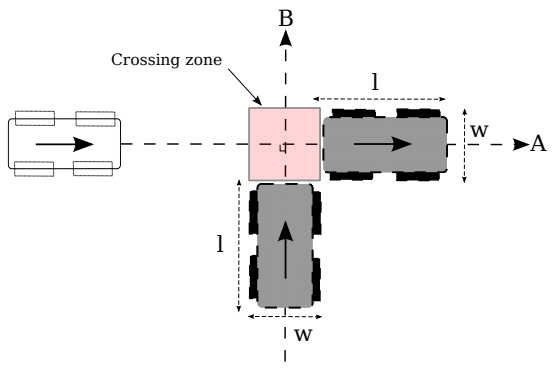

Fig. 2: Crossing zone with an angle $\theta=90^{\circ}$

2) $\theta<90^{\circ}$ : In this case, one vehicle can enter the (diamond-shaped) crossing zone before the previous vehicle has completely left (see Figure 3). To minimize the time between two vehicles, the crossing zone must be shared by both vehicles from each flow.

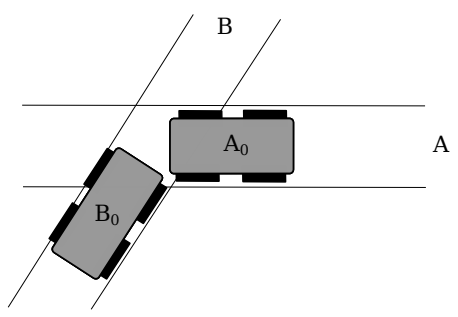

Fig. 3: Shared zone when $\theta<90^{\circ}$

We deduce the minimum period for passing two vehicles from each flow (see details in [8, Appendix]):

$$
T=\frac{2\left(w \frac{(1-\cos \theta)}{\sin \theta}+l\right)}{V} .
$$

3) $\theta>90^{\circ}$ : In this case, there is no (simultaneous) sharing of the crossing zone between vehicles from the two flows. The minimum period for passing two vehicles from each flow is 
(see details in [8, Appendix]):

$$
T=\frac{2\left(w \frac{(1+\cos \theta)}{\sin \theta}+l\right)}{V} .
$$

4) Comments -: $T$ has been determined assuming the maximum possible flows of vehicle. But, if less vehicles are going through the intersection, this just implies leaving "unused" intervals. Also, in practice, $T$ will be increased by $\epsilon$, a safety margin of vehicle inter-distance.

\section{Temporal Synchronization of 2 Roads}

1) Principle - : Knowing the minimal period $T$, we organize the passage of vehicles each half-period $T_{c}=T / 2$, i.e., one road passing one vehicle at even half-periods (even multiples of $T_{c}$ ), while the odd half-periods correspond to the other road. Figure 4 illustrates this alternating principle of period $T_{c}$.

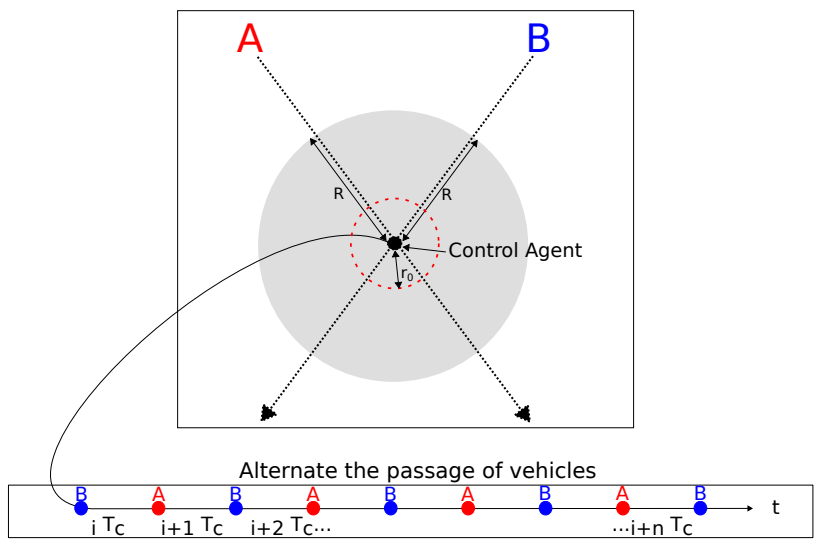

Fig. 4: Synchronization principle of an intersection

One issue is to ensure that the vehicle agents arrive in crossing zones at the default speed $V$ and at the right time to be synchronized as desired. This requires a specific synchronization phase prior to each crossing zone. To that end, we define two radii from the center of an intersection:

- $\quad r_{0}(>0)$ the distance within which a vehicle is required to run at the default speed $V$; and

- $\quad R\left(>r_{0}\right)$ the distance within which an incoming vehicle should adapt its speed to synchronize with the intersection (speed changes occur between $R$ and $r_{0}$ before the center).

This defines a specific control zone for an intersection.

For each vehicle, the synchronization requires computing (1) at which half period of the crossing zone to pass, and (2) how to adapt its speed to be synchronized. As a vehicle agent may not know exactly when to pass, in particular if there could be conflicts with other vehicles in the same lane, we need to introduce a control agent at each intersection, which is in charge of determining when each incoming vehicle agent should pass, and thus needs perceiving and communicating with vehicles within range $R$.
2) Synchronizing Vehicles with an Intersection -: A first observation is that each lane can be handled independently. Note also that a vehicle could arrive earlier or later than its originally predicted arrival time. Yet, rather than searching for the solution optimizing delays or energy consumption, we decide here to slow down all vehicles.

Considering vehicle agent $i$ entering a control zone at current time $t$ and at default velocity $V$, its predicted crossing time at the intersection is $t_{a}=t+R / V$ (forgetting about previous vehicles). The closest next half-period is numbered $n_{i}=\left\lceil t_{a} / T-p / 2\right\rceil$, where $p=0$ or 1 depending whether $i$ is on an even or odd road. Knowing that the last used halfperiod (by previous vehicles) on this lane is numbered $n_{\text {last }}$, if $n_{\text {last }} \geq n_{i}$, then $n_{i}$ should be set to $n_{\text {last }}+1$.

Then, to ensure that vehicle agent $i$ enters the crossing zone at the right time and speed so as to cross the intersection at time $n_{i} T+p T_{c}$, it should follow a speed profile such as presented on Fig. 5, where the vehicle (1) slows down (acceleration $a_{1}<0$ ), (2) then runs at constant speed $V_{x}$, and (3) finally speeds up (acceleration $a_{2}>0$ ).

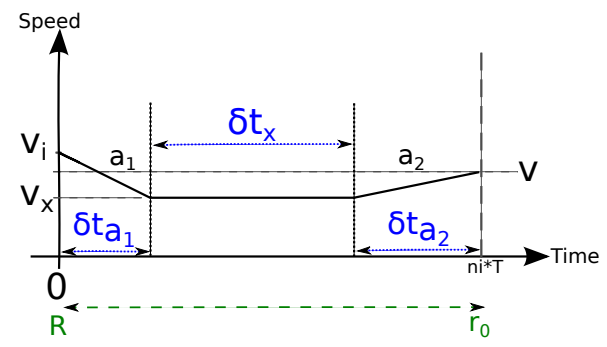

Fig. 5: Speed profile

The other constraints for this profile are:

- the speed is $V_{i}$ at the start $(\neq V$ if the vehicle has been slowed down by a vehicle in front of him) and $V$ at the end (as required);

- $\quad$ the total length is $R-r_{0}$; and

- the total duration is $\left(n_{i} T+p T_{c}-r_{0} / V\right)-t$.

In our simplified model, using the maximum acceleration $\left(a_{2}\right)$ and deceleration $\left(a_{1}\right)$ of the vehicle allows minimizing the loss of kinetic energy.

Solving this problem leads to solving the following quadratic equation:

$$
\begin{aligned}
& V_{x}^{2}\left(\frac{a_{1}-a_{2}}{2 a_{1} a_{2}}\right)+V_{x}\left(\left(t_{p}-t\right)+\frac{a_{2} V_{i}-a_{1} V}{a_{1} a_{2}}\right) \\
& -\left(r_{i}-r_{0}\right)-\frac{a_{2} V_{i}^{2}-a_{1} V^{2}}{2 a_{1} a_{2}}=0 .
\end{aligned}
$$

A solution exists only if there is enough space to slow down the vehicle agent as desired. Moreover, we should verify first if there exist mathematically feasible solutions $(\Delta \geq 0)$, then the physical feasibility of these solutions, i.e., that the resulting speeds, distances and durations in the speed profile are all positive. 
3) Algorithm - : Algorithm 1 thus shows how the control agent handles a single lane, whose parity is known through a parameter $p \in\{0,1\}$. The algorithm computes for each vehicle its programmed arrival time $t_{p}$ at the crossing zone, and the related speed profile (recomputing it regularly to adapt to unexpected delays). Note also that $n_{i}$ should be computed only the first time vehicle $i$ enters the control zone. Otherwise, due to small errors, the value $n_{i}$ could change from one iteration to the next. This is achieved by assigning a default value of $n_{i}=-1$ when vehicle $i$ is first detected by the control agent, having the control agent remember $n_{i}$ from one iteration to the next, and computing $n_{i}$ if and only if $n_{i}=-1$ (line 6).

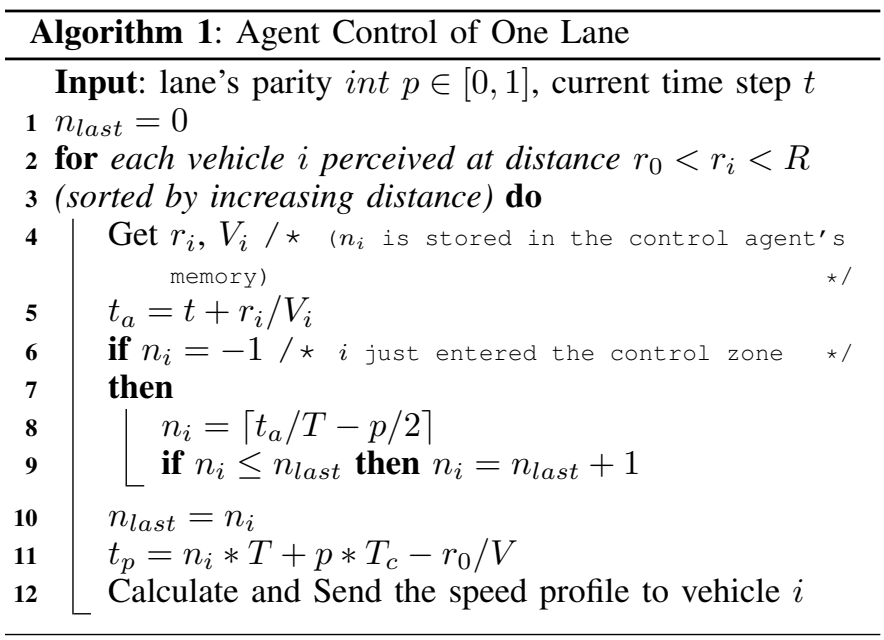

4) Minimum Radii — : The radii $r_{0}$ and $R$ should not be taken arbitrarily.

$r_{0}$ should be such that, when the center of vehicle $i$ is at distance $r_{0}$ from the center of the intersection, then the nose of $i$ has not yet entered the crossing zone. This implies

$$
r_{0} \geq \frac{l}{2}+\frac{w \max \left(\cos \frac{\theta}{2}, \cos \frac{\pi-\theta}{2}\right)}{\sin \theta}
$$

(see details in [8, Appendix]).

$R$ should be such that the quadratic equation can always be solved (satisfying the aforementioned mathematical and physical constraints) For a single vehicle, the limit case is when it just misses a period, and should thus wait for the next period, i.e., the duration of the speed profile will be $t_{p}-t=\frac{R-r_{0}}{V}+T+\epsilon$ instead of $\frac{R-r_{0}}{V}+\epsilon$. This problem becomes more complex when multiple vehicles have to queue. Indeed, there is no ideal solution for $R$ because there can always be vehicles arriving in overly large numbers. In practice (see experiments) we ensure that the radius $R$ is large enough to allow the application of the speed profile, but check that this radius remains reasonable.

\section{Road with Two Opposite Lanes}

In this section we briefly show how to adapt the solution presented in the previous sections to the case of two roads each made of two opposite lanes, i.e., the case -shown on Figure 1 (right)- we will consider in our experiments.

First, due to symmetries, vehicles from the same road should go through the crossing zone simultaneously, i.e.,

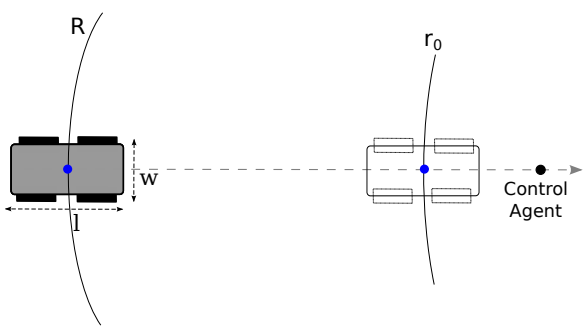

Fig. 6: Radii $R$ and $r_{0}$

two vehicles from lanes $a$ and $c$ pass simultaneously at the intersection, then two vehicles from $b$ and $d$, and so on.

The minimum period $T$ for crossing must be adjusted to this specific case to account for (i) the size of the new crossing zone, and (ii) the synchronization pattern the vehicles should follow. Let $\lambda$ be the distance separating two opposite lanes of the same road (see Fig. 7). The minimum period is (see details in $[8$, Appendix]):

$$
T=\frac{2\left(\frac{2\left(w+\frac{\lambda}{2}\right)}{\sin \theta}+l\right)}{V} .
$$

Furthermore, we should adapt the radius $r_{0}$ to this configuration. Thus, instead of using the width $w$ of a vehicle as the width of the road, we will consider the width of a two-lane road.

The control agent's algorithm is unchanged, each lane being handled independently of the other lanes, e.g., each lane having its own $n_{\text {last }}$ variable. One should just pay attention to the fact that, for a given lane, distances are not computed with respect to the center of the crossing zone, but with respect to the middle of the lane's segment inside the crossing zone, as illustrated on Figure 7.

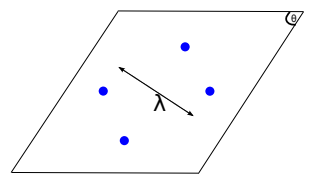

Fig. 7: Crossing zone in the case of 2 opposite lanes in each road

\section{EXPERIMENTAL RESULTS}

In this section we evaluate our approach on a network of three roads organized as a triangle, giving results for a single intersection between two two-lane roads, as in Figure 8, before considering more complex networks.

We compare our approach to a strategy based on traffic lights with a fixed time for each cycle. A traffic light is placed at the entrance of each flow of the intersection. The lights can switch the passage of vehicles between two roads, for fixed (equal) periods.

\section{A. Simulations}

We have developed (in JAVA) a continuous-space and discrete-time simulator of a network of roads. 


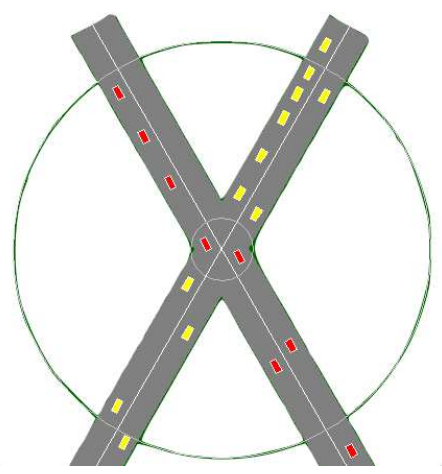

Fig. 8: Simulator : illustration of an intersection control

The detailed experimental setting for our three-road network is the following:

- roads are $1000 \mathrm{~m}$ long and the angle of the intersection is $\frac{\pi}{3}$;

- the control agents' range of action $R$ is $200 m$ and $r_{0}=30 m$;

- the maximum speed of each vehicle is $10 \mathrm{~m} / \mathrm{s}$, the maximum acceleration is $1.5 \mathrm{~m} / \mathrm{s}^{2}$ and the maximum deceleration is $-1.5 \mathrm{~m} / \mathrm{s}^{2}$;

- we used a near-to-near longitudinal control developed in [9], which ensures a collision-free behavior between the same lane vehicles (only outside the control zone);

- $\quad$ at each entrance of the network, we installed a source that generates vehicles following a Bernoulli distribution with parameter $\frac{1}{D}$ ( $D$ is the average time, in seconds, between two consecutive injections);

- the vehicles have dimension $l=5 m, w=2.5 m$ and safety margin of vehicle inter-distance $\epsilon=1.5 \mathrm{~m}$;

- Simulation time step is set to $0.1 s$.

In this case, the minimum period is $T=3.2 s$.

For the traffic lights approach, we can fix the green and red times for each flow. We vary their duration in the following subsection but the reference value is equal to $30 \mathrm{~s}$ for each color. The green lights of the same road are turned on at the same time.

\section{B. Comparing Various Strategies}

Our objective is to compare our approach with traffic lights by observing the resulting delays -i.e., the difference between the theoretical and actual traversal times- when 100 vehicles traverse the network under a high injection frequency $(D=$ $4 s$ ), i.e., heavy traffic.

Figure 9 presents simulation results for our algorithm and traffic lights with different durations $(10 s, 20 s, 30 s)$. The $\mathrm{X}$ axis represents the number of vehicles having left the network in their output order and the $\mathrm{Y}$ axis gives the vehicle's average delay in seconds (plus standard deviation) over 100 simulations.

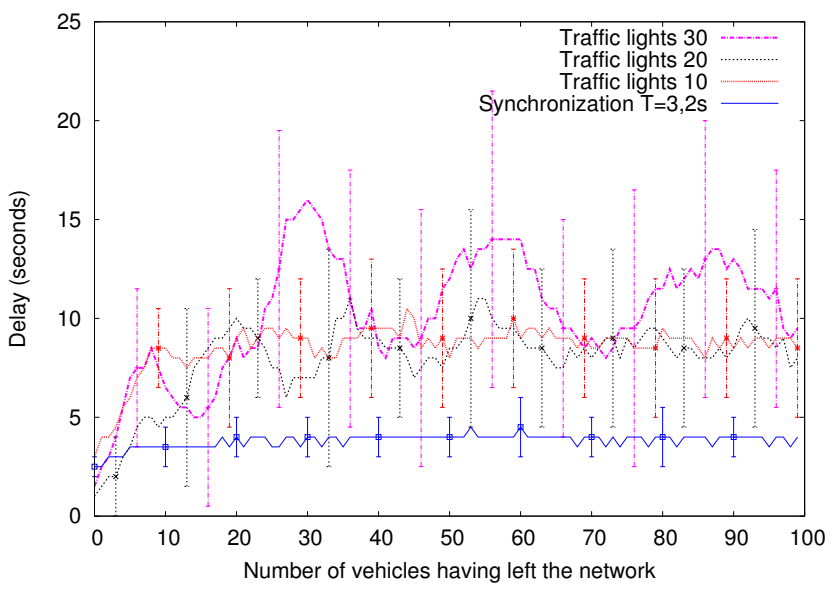

Fig. 9: Synchronization vs. Traffic lights

Our approach is clearly more efficient in this experiment compared to traffic lights. Here, the worst delay produced by a vehicle with the local synchronization approach does not exceed $6 s$, while for traffic lights it exceeds $20 s$ (maximum value with the standard deviations using $30 s$ duration). The average delay is below $4 s$ for the local synchronization while, for the best traffic lights curve, it is around $9 s$ (using $10 s$ or $20 s$ duration). We also tested the traffic lights strategy with less than $10 \mathrm{~s}$ duration and we observed that the average delay increases significantly. This is mainly due to vehicles requiring more time to traverse an intersection when they have to restart, which even leads to queue formations and collisions at or below $5 s$.

\section{Effect of Varying $R$}

In this section, we measure the effect of varying the range of action $R$ of the control agent to evaluate whether this influences the quality of our solution (without modifying $r_{0}$ ). Table I gives the averages and the standard deviations of the energy consumed when $R=50 \mathrm{~m}, R=100 \mathrm{~m}$ and $R=200 \mathrm{~m}$. This consumed energy is measured through the total change in velocity during the travel of 1000 vehicles. We observe that the delays do not change significantly, but there is a difference in terms of energy consumed when we reduce the crossing zone. It increases when we use a small $R$. This is due to the small distance that forces the control agent to slow down the vehicles suddenly.

TABLE I: Averages and Standard Deviations of the Energy Consumed

\begin{tabular}{|c|c|c|c|}
\hline$D=10 \mathrm{~s}$ & $50 \mathrm{~m}$ & $100 \mathrm{~m}$ & $200 \mathrm{~m}$ \\
\hline \hline Average Energy & 8.6 & 5.2 & 4 \\
\hline Standard Deviation & \pm 3.1 & \pm 3.84 & \pm 4.6 \\
\hline
\end{tabular}

\section{Effect of Varying $D$}

We want to see here if our approach is efficient when the vehicles' throughput is higher (generally the reservation based approaches, as in [1], have poor resistance). Figure 10 
shows the variations of delays depending on the injection throughputs. The plotted histograms are averages of 10000 vehicles. We used $\mathrm{D}$ with the values $1 s, 2 s, 3 s, 4 s$ and $6 s$ which correspond respectively to the throughputs 60,30 , 20,15 and 10 vehicles/minute for each source. According to the figure, the average delay increases slightly (less than $1 s$ ) when the throughput is less than the minimal period of the intersection $(3.2 \mathrm{~s})$. Immediately thereafter (using $30 \mathrm{veh} / \mathrm{m}$ and $60 \mathrm{veh} / \mathrm{m}$ ) we saturate the system, as also observed by [1].

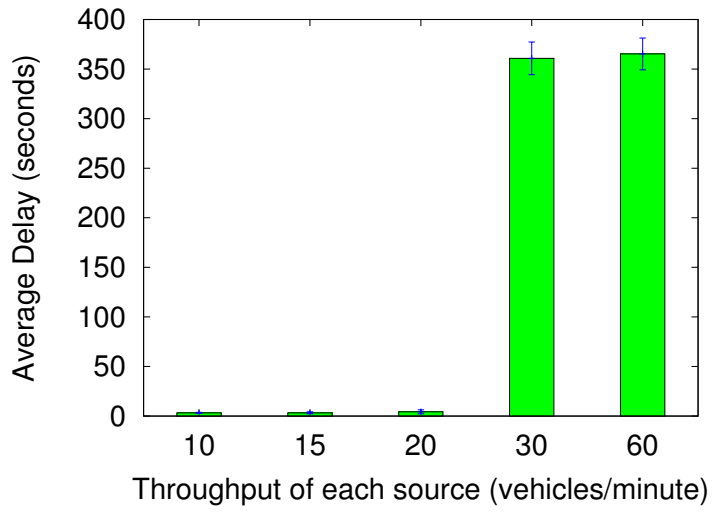

Fig. 10: Throughput variation

\section{E. Increasing the number of intersections}

We tested our approach on a network of three two-lane roads with five intersections (Figure 11). Note that intersections are independent, each with its own angle-dependent period. The measurements on this network give equivalent gains to one intersection. The approach appears scalable as a consequence of its decentralized nature. We verified in all our simulations that we never have collisions between vehicles. A video showing the simulator can be viewed at http://www.loria.fr/\% 7Emtlig/\#Videos.

Future works will be dedicated to the network level, by focusing on the question of synchronization between control agents. This involves looking for synchronization relationships between the agents linked by the same road, and how they can cooperate to further optimize the traffic.

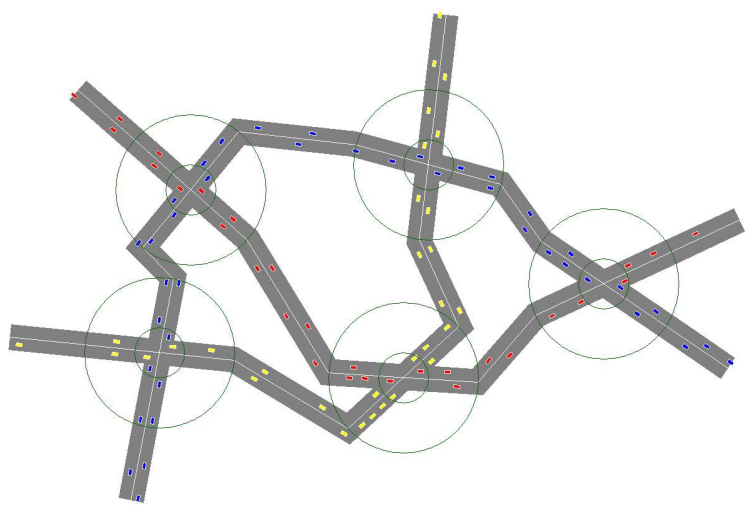

Fig. 11: Illustration of 5 intersections control

\section{CONCLUSION}

In this paper we addressed the problem of automatically controlling vehicle crossings on a network, or more generally of mobile agents (e.g., robots). To that end, we proposed a strategy based on a local control of vehicles at intersections. It consists in spacing vehicles such that they can cross without being stopped. By synchronizing the arrival of vehicles with a similar period, but phase-shifted by a half period, vehicles from both flows pass alternately. Therefore the vehicles potentially face a slight slow-down in order to be able to cross the intersection, which is better than stopping them.

Based on this principle, we defined a crossroad/intersection agent at each intersection which uses only its local perceptions of the traffic. It determines the instructions for each vehicle to cross the intersection based on its distance and on the parity assigned to the road. The experimental study demonstrated the ability to regulate the traffic at intersections, and the significant gain in terms of time compared to a conventional traffic lights system.

Future work includes first continuing the experimental study to test the limits of our approach and further evaluate it in a wide variety of scenarios. The other perspective that motivates our research is to let the control agents communicate and synchronize with each other in order to further improve the traffic on the network, typically by inducing green waves. As these synchronization constraints involve neighboring intersections, locally interacting control agents could find a globally efficient solution.

\section{REFERENCES}

[1] K. Dresner and P. Stone, "Multiagent traffic management: A reservationbased intersection control mechanism," in Proceedings of the Third International Joint Conference on Autonomous Agents and Multiagent Systems (AAMAS), 2004.

[2] C. Fok, M. Hanna, S. Gee, T. Au, P. Stone, C. Julien, and S. Vishwanath, "A platform for evaluating autonomous intersection management policies," in Third International Conference on Cyber-Physical Systems (ICCPS). IEEE, 2012, pp. 87-96.

[3] N. Bhouri, F. Balbo, S. Pinson, and M. Tlig, "Collaborative agents for modeling traffic regulation systems," in Web Intelligence and Intelligent Agent Technology (WI-IAT), 2011 IEEE/WIC/ACM International Conference on, vol. 2, aug. 2011, pp. $7-13$.

[4] N. Hounsell and B. Shrestha, "A new approach for co-operative bus priority at traffic signals," Intelligent Transportation Systems, vol. 13, no. 1 , pp. $6-14,2012$.

[5] K. Dresner and P. Stone, "Multiagent traffic management: An improved intersection control mechanism," in Proceedings of the Fourth International Joint Conference on Autonomous Agents and Multiagent Systems (AAMAS), 2005.

[6] R. Naumann and R. Rasche, Intersection collision avoidance by means of decentralized security and communication management of autonomous vehicles. Univ.-GH, SFB 376, 1997.

[7] R. Naumann, R. Rasche, J. Tacken, and C. Tahedi, "Validation and simulation of a decentralized intersection collision avoidance algorithm," in Intelligent Transportation System (ITSC'97), IEEE, 1997, pp. 818-823.

[8] M. Tlig, O. buffet, and O. Simonin, "Decentralized traffic management: A synchronization-based intersection control - extended version," Tech. Rep., 2014, http://hal.inria.fr/hal-00960735.

[9] A. Scheuer, O. Simonin, and F. Charpillet, "Safe longitudinal platoons of vehicles without communication," in Proceedings of the international conference on Robotics and Automation (ICRA), 2009, pp. 2835-2840. 